\title{
Finally, some signaling molecules find a home in yeast
}

\author{
Steven J. Isakoff, Yanli Wang, and Edward Y. Skolnik
}

In this issue of Nature Biotechnology, SupertiFurga et al. ${ }^{1}$ describe an approach to identifying molecules that negatively regulates the tyrosine kinase Src. Src is a cytoplasmic tyrosine kinase that, among its many functions, mediates cell growth and differentiation. The Src family of tyrosine kinases is not expressed in the fission yeast Schizosaccharomyces pombe. These investigators have previously demonstrated that expression of c-Src in S. pombe is lethal ${ }^{2}$. The mechanism whereby Src induces lethality in $S$. pombe is not known. However, the finding that catalytically inactive Src is not lethal when expressed in $S$. pombe, together with the finding that wildtype $c$-Src induces tyrosine phosphorylation of a variety of cellular proteins, suggests that c-Src kills S. pombe by stimulating aberrant tyrosine phosphorylation of one or more critical protein targets.

Using the suppression of Src lethality as a biological endpoint, the investigators previously used this system to study the negative regulation of Src by Csk, a protein tyrosine kinase that negatively regulates Src by phosphorylating Src in its C-terminus on tyrosine-5272 Thus, the stage was set to determine whether novel molecules capable of inhibiting the Src tyrosine kinase could be identified by transfecting S. pombe engineered to express Src under an inducible promoter with human cDNA libraries. Using this approach, SupertiFurga et al. have identified two classes of molecules that rescue Src lethality in S. pombe. The first class consists of Csk. While this finding in itself is not novel, it validates the approach by demonstrating that specific inhibitors of Src family tyrosine kinases can be identified using this screen. The second class of molecules consists of three different protein tyrosine phosphatases (PTPs). In contrast to Csk, the ability of PTPs to inhibit Src is unlikely to be specific to a particular phosphatase. Rather, as pointed out by the investigators, a variety of PTPs suppress the lethality of c-Src by either dephosphorylating the $\mathrm{c}$-Src substrates, or by dephosphorylating the tyrosine-416 on Src that is required for the full activation of the $\mathrm{c}$ Src kinase.

At first glance, it may appear that this approach has added little to our understanding of the regulation of $\mathrm{c}-\mathrm{Src}$ beyond what was already known, since no novel Src inhibitors

Steven J. Isakoff, Yanli Wang, and Edward Y. Skolnik are at the department of pharmacology, Skirball Institute for Biomolecular Medicine, New York University Medical Center, New York, NY 10016

(edward.skolnik@moska.med.nyu.edu). were identified. In addition, because this is not a true genetic screen, some of the same limitations encountered using more traditional biochemical methods apply. In particular, the finding that a variety of different tyrosine phosphatases are capable of rescuing Src lethality indicates that this approach, when used by itself, is unlikely to identify the physiologically relevant tyrosine phosphatase responsible for negatively regulating Src in mammalian cells. Another potential problem, although one that is amenable to modification of the procedure, is that all of the negative regulators identified

\section{Despite its limitations, this} approach is very powerful and offers several advantages over the more standard biochemical and molecular approaches that have been used to identify inhibitors of tyrosine

\section{kinases. It will lend itself to a variety of applications.}

are enzymes. While this may indicate a true lack of an allosteric Src regulator, allosteric inhibitors of other tyrosine kinases have been identified ${ }^{4}$. It is more likely that the inability to identify allosteric inhibitors reflects the different levels of expression from the library and of c-Src. As discussed by the investigators, expression of cDNA libraries using stronger yeast promoters may allow allosteric regulators of Src to be identified by this method.

Despite its limitations, this approach is very powerful and offers several advantages over the more standard biochemical and molecular approaches that have been used to identify inhibitors of tyrosine kinases. The method will likely lend itself to a variety of applications outside those presented in the current report. One major advantage is the relative ease with which the molecules can be identified. Conventional biochemical approaches used to clone and isolate Csk are arduous and require the use of large quantities of tissue to purify sufficient protein for microsequence analysis and subsequent cDNA cloning ${ }^{3}$. Tyrosine kinases other than Src cause lethality in S. pombe, and should be amenable to similar screens ${ }^{1,5}$. The fact that most new PTPs were previously identified by PCR and low stringency hybridization screen- ing suggests that the functional screen described here may allow identification of new PTPs that are not amenable to identification using the other approaches. In addition, specific nonPTP inhibitors of a particular subgroup of tyrosine kinases may also be identified in these screens. For example, proteins that interact with, and negatively regulate, the tyrosine kinase $\mathrm{Abl}$ have been biochemically characterized $^{6}$. The finding that $\mathrm{Abl}$ is lethal when expressed in S. pombe suggests that these proteins may be cloned by using the approach described by Superti-Furga et al.

Their method should be easily amenable to automation, providing an initial assay for the identification of novel pharmaceutical inhibitors of the enzymes. A tyrosine kinase inhibitor should rescue $S$. pombe from the lethal effects of Src. Inhibitors of PTPs could be identified by determining whether a compound restores the lethal effect of Src in cells expressing both Src and a PTP. The use of a reliable cell-based assay is a welcome addition to the cell-free biochemical assays that have previously been used to screen for inhibitors of PTPs.

Another exciting possibility is that the same approach for identifying inhibitors of tyrosine kinases could be applied to the hunt for inhibitors of other classes of molecules. Several other enzymes, such as the p110 catalytic subunit of mammalian phosphatidylinositol (PI) 3-kinase and some isoforms of protein kinase $\mathrm{C}$ are also lethal to $S$. pombe when expressed at high levels,

PI3-kinase is a lipid kinase that is responsible for phosphorylating the 3-OH group of phosphatidylinositol leading to the generation of PI $(3,4,5)$ P3 ${ }^{10}$. PI $(3,4,5)$ P3 functions directly as a second messenger to mediate a variety of biological responses including cell growth and membrane trafficking. However, little is known about the molecules that negatively regulate this pathway. The screen described by SupertiFurga et al. may provide a powerful tool to uncover proteins that regulate these and other molecules.

\footnotetext{
1. Superti-Furga, G., Jönsson, K., and Courtneidge, S.A 1996. Nature Biotechnology14:600-605.

2. Superti-Furga, G. et al. 1993. EMBO J. 12:2625-2634.

3. Nada, S. et al. 1991. Nature 351:69-72.

4. Maddux, B.A et al, 1995. Nature 373:384-385.

5. Walkenhorst, J., Goga, A., Witte, O.N., and SupertiFurga, G. Oncogene (In press).

6. Pendergast, A.M. et al. 1991. Proc. Natl. Acad. Sci. USA 88:5927-5931.

7. Mayer, B. and Baltimore, D. 1994. Mol. Cell. Biol. 14:2883-2894.

8. Kodaki, T. et al. 1994. Eur. J. Biochem. 219:775-780.

9. Goode, N.T., Hajibagheri, M.A., Warren, G., and Parker, P.J. 1994. Mol. Bio. Cell. 5:907-920.

10. Cantley, L.C. et al. 1991. Cell 64:281-302.
} 